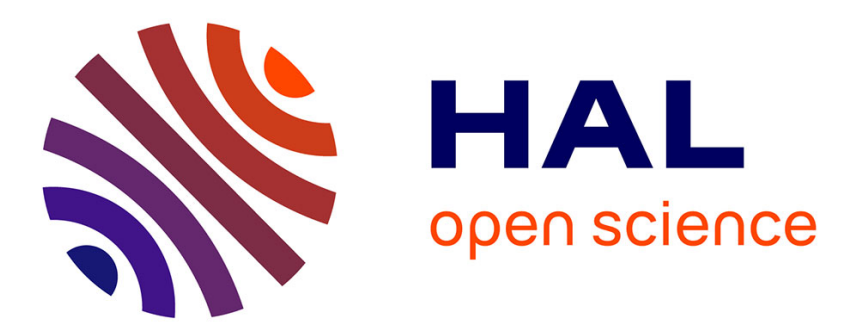

\title{
Spondyloarthrite et sarcoïdose : faux amis ou véritable association? Revue systématique de la littérature
}

S. Cadiou, F. Robin, R. Guillin, A. Perdriger, S. Jouneau, N. Belhomme, G. Coiffier, P. Guggenbuhl

\section{- To cite this version:}

S. Cadiou, F. Robin, R. Guillin, A. Perdriger, S. Jouneau, et al.. Spondyloarthrite et sarcoïdose : faux amis ou véritable association? Revue systématique de la littérature. Revue du Rhumatisme, 2021, 88 (2), pp.101-109. 10.1016/j.rhum.2021.01.015 . hal-03196157

\section{HAL Id: hal-03196157 https://hal.science/hal-03196157}

Submitted on 13 Apr 2021

HAL is a multi-disciplinary open access archive for the deposit and dissemination of scientific research documents, whether they are published or not. The documents may come from teaching and research institutions in France or abroad, or from public or private research centers.
L'archive ouverte pluridisciplinaire $\mathbf{H A L}$, est destinée au dépôt et à la diffusion de documents scientifiques de niveau recherche, publiés ou non, émanant des établissements d'enseignement et de recherche français ou étrangers, des laboratoires publics ou privés. 
Spondyloarthrite et sarcoïdose : faux amis ou véritable association ? Revue systématique de la littérature

Cadiou S (1), Robin F (1), Guillin R (2), Perdriger A (1), Jouneau S (3), Belhomme N (4), Coiffier G (1), and Guggenbuhl $P(1,5)$.

(1) Department of Rheumatology, Rennes University Hospital, 35000, Rennes, France.

(2) Department of Medical Imaging, Rennes University Hospital, 35000, Rennes, France.

(3) Department of Respiratory Medicine, Rennes University Hospital, Rennes, France. University of Rennes 1, Rennes, France. INSERM-IRSET UMR1085, Rennes, France.

(4) Internal Medicine Department, Rennes University Hospital, 35000, Rennes, France.

(5) CHU Rennes, Univ Rennes, INSERM, Institut NUMECAN (Nutrition Metabolisms and Cancer), F35000, Rennes, France. UMR INSERM U 1241, University of Rennes 1, 35000, Rennes, France.

Auteur correspondant : Simon Cadiou sicadiou@gmail.com

\section{Résumé}

Contexte : sarcoïdose et spondyloarthrite $(\mathrm{SpA})$ sont fréquemment associées, et des granulomes ont été décrits au niveau des os iliaques. Nous proposons ici une revue systématique de la littérature sur les manifestations rhumatologiques axiales de la sarcoïdose.

Méthodes: cette revue systématique de la littérature a été réalisée dans les bases de données PubMed et Cochrane Library. Les recommandations PRISMA (Preferred Reporting Items for Systematic Reviews and Meta-Analyses) ont été appliquées pour l'examen des rapports de cas et des études transversales.

Résultats : au total, 41 articles remplissaient les critères définis. Trois études transversales sur l'association SpA-sarcoïdose ont montré une prévalence de sacro-iliite et de SpA de 12,9 à 44,8 \% et de 12,9 à 48,3\%, respectivement, dans les sous-groupes de lombalgie inflammatoire (LI). Cependant, les définitions de la LI et les modalités d'imagerie de l'articulation sacro-iliaque (ASI) étaient hétérogènes (radiographie ou imagerie par résonance magnétique), la radiographie étant la principale technique (78 \% des cas) employée pour diagnostiquer la sacro-iliite. Trente-et-un rapports de cas sur l'association sarcoïdose-sacro-iliite ont été identifiés, représentant 35 patients. Les critères de l'ASAS pour la SpA étaient remplis dans la moitié des cas (16/32), et le HLA-B27 était positif dans $46 \%$ des cas (12/26). La sarcoïdose est apparue postérieurement aux symptômes sacro-iliaques chez 47 \% des patients. L'atteinte unilatérale était significativement plus fréquente dans les sept cas de granulome des os sacro-iliaques que dans le groupe sarcoïdose-sacro-iliite $(p<0,001)$.

Conclusion: l'analyse de la littérature a démontré de façon probante l'existence d'une association SpA-sarcoïdose, ce qui incite à accorder une attention particulière aux patients atteints de sarcoïdose et faisant état d'une LI. La présence d'une sacro-iliite unilatérale devrait faire suspecter une atteinte osseuse granulomateuse distincte de la sacro-iliite. Les modalités d'imagerie mises en œuvre pour étudier l'ASI chez les patients présentant une sarcoïdose étaient hétérogènes et des études supplémentaires sont nécessaires. 
Mots clés : sarcoïdose, sacro-iliite, articulation sacro-iliaque, spondyloarthrite, os, granulome 


\section{Introduction}

La sarcoïdose est une affection granulomateuse d'origine inconnue [1]. Les poumons et les ganglions lymphatiques médiastinaux sont fréquemment touchés mais des granulomes peuvent être observés dans tous les organes, et plus particulièrement la peau, le système lymphatique, les yeux et le foie [1]. Le système musculosquelettique est concerné chez 4 à $38 \%$ des patients [2], sous des formes aiguës comme le syndrome de Löfgren ou chroniques comme l'arthropathie de la sarcoïdose, avec granulome sarcoïdien dans la membrane synoviale. La sarcoïdose osseuse est une atteinte rare qui touche 3 à $13 \%$ des patients présentant une sarcoïdose [3]. La sacro-iliite dans la sarcoïdose a été décrite pour la première fois en 1951 dans un rapport de cas de Verstraeten et al. [4]. La sacroiliite se caractérise par une inflammation de l'ASI et une lombalgie souvent d'horaire inflammatoire. Depuis la publication de cet article, des liens entre sarcoïdose et sacro-iliite ont régulièrement été mis en avant dans la littérature. La majorité des études décrivent une sacro-iliite ou une atteinte des os iliaques visible à la radiographie, mais dans un nombre limité de cas [5,6].

L'objectif de cette revue systématique était d'identifier les caractéristiques diagnostiques, y compris les critères radiologiques, de la sacro-iliite chez les patients présentant une sarcoïdose avec atteinte sacro-iliaque. Nous avons inclus des rapports de cas et des études prospectives et rétrospectives.

\section{Méthodes}

a. Recherche de publications

Cette revue systématique a été menée en janvier 2020 dans les bases de données Medline (via PubMed) et Cochrane. Nous avons identifié des articles en anglais et en français en appliquant la méthode PRISMA [7]. Les mots clés de recherche utilisés étaient (sarcoidosis OR sarcoid OR sarcoid*) AND (sacroiliitis OR sacroiliac OR ankylosing spondylitis OR spondyloarthritis OR spondyloarthropathy). D'autres articles pertinents ont été identifiés dans d'autres sources, essentiellement dans les références bibliographiques des articles présélectionnés.

\section{b. Critères d'inclusion}

Tous les modèles de conception (rapport de cas, étude rétrospective ou prospective, étude transversale) ont été inclus. Après extraction des doublons, nous avons sélectionné les articles évaluant les ASI de patients atteints de sarcoïdose. Le diagnostic de sarcoïdose devait être confirmé par une analyse histologique montrant des granulomes non caséeux et par l'exclusion d'autres maladies granulomateuses, en particulier les infections mycobactériennes [8]. Nous n'avons pas inclus les réactions granulomateuses à la prise d'inhibiteurs du facteur de nécrose tumorale alpha (anti-TNFa) pour traiter la spondyloarthrite (SpA) ou d'autres maladies auto-immunes en raison du lien iatrogène des réactions médicamenteuses de type sarcoïdose (RMTS) [9] consécutives à un traitement par antiTNFa et car l'immunopathogenèse de la sarcoïdose est mal connue. Les RMTS dues aux anti-TNFa sont considérées comme un diagnostic différentiel compte tenu de la réaction médicamenteuse granulomateuse mentionnée dans la déclaration de l'ATS/ERS/WASOG sur la sarcoïdose [8].

\section{c. Collecte des données}

Nous avons collecté les données suivantes dans les rapports de cas : sexe, âge au moment du diagnostic, imagerie utilisée par les auteurs pour diagnostiquer la sacro-iliite, présence de sacro-iliite selon les critères de l'ASAS de 2009 [10], score radiographique des ASI selon les critères de New York 
modifiés (NYm), ankylose, statut HLA-B27, présence de lombalgie (mécanique ou inflammatoire), lombalgie depuis au moins 3 mois, apparition de la maladie avant 45 ans, caractéristiques de la SpA (LI, arthrite, enthésite, dactylite, uvéite, psoriasis, maladie inflammatoire chronique de l'intestin, bonne réponse aux AINS, antécédents familiaux de SpA, CRP élevée), diagnostic de sarcoïdose (granulomes révélés par une biopsie et/ou syndrome de Löfgren), classification de la sarcoïdose sur une radiographie thoracique, organes touchés par le granulome et relation chronologique entre la sarcoïdose et les symptômes des ASI (diagnostic antérieur, postérieur ou simultané).

\section{Résultats}

Au total, nous avons présélectionné 319 articles dans PubMed et 37 dans Cochrane Library. Après élimination des doublons et ajout de cinq articles identifiés dans les références bibliographiques des publications présélectionnées, 335 articles ont été sélectionnés. La lecture du titre et du résumé a permis d'exclure 267 articles et d'évaluer l'éligibilité du texte complet de 70 autres. Vingt-neuf articles complets ont été exclus, la plupart en raison de RMTS consécutives à un traitement anti-TNFa. Enfin, 41 articles ont été retenus pour l'étude. Le diagramme des flux PRISMA ${ }^{\odot}$ est présenté à la figure 1. Trois des 41 articles sélectionnés étaient des études transversales [5,11,12] (résumées dans le tableau 1). Deux groupes ont été définis à partir des 38 rapports de cas : un groupe sarcoïdose-sacroiliite [4,13-42] et un groupe d'atteinte granulomateuse des os sacro-iliaques imitant la sacro-iliite [6,4348]. Les principales caractéristiques du groupe sarcoïdose-sacro-iliite sont résumées dans les tableaux 2 et 3 et celles du groupe atteinte osseuse sacro-iliaque dans le tableau 4.

a. Études transversales

En 2005, Erb et al. [11] ont publié la première étude de cohorte monocentrique transversale sur la lombalgie analysant 61 patients atteints de sarcoïdose. La quasi-totalité des patients était de type caucasien, avec un rapport des sexes proche de 1. Cinq patients ne remplissaient pas les critères de I'ATS/ERS/WASOG pour la sarcoïdose en raison de l'absence de résultats histologiques compatibles ou de syndrome de Löfgren, mais ont néanmoins été considérés comme atteints de sarcoïdose et inclus dans l'étude. L'évaluation radiographique des ASI a été effectuée par une double lecture mais les critères de diagnostic de la sacro-iliite n'étaient pas mentionnés. Les auteurs ont considéré que quatre patients présentaient une sacro-iliite, bilatérale dans un seul cas. Cette étude a analysé la sacro-iliite indépendamment du diagnostic de SpA mais un seul patient présentait l'antigène HLA-B27. Aucune différence n'a été observée entre les patients avec et sans sacro-iliite. La prévalence de la sacro-iliite a été estimée à 6,6\% (4/61) dans le groupe sarcoïdose, 8,2 \% (4/49) dans le sous-groupe lombalgie et $12,9 \%(4 / 31)$ dans le sous-groupe LI.

Kobak et al. [5] ont décrit six cas de sacro-iliite bilatérale selon les critères NYm à partir de radiographies pelviennes standard d'une cohorte monocentrique de 42 patients présentant une sarcoïdose confirmée par biopsie. Tous les patients du groupe sacro-iliite radiographique présentaient une LI selon les critères de Calin. Les auteurs ont diagnostiqué six cas de SpA selon les critères de l'ASAS 2009 [10]. Les patients du groupe SpA-sarcoïdose étaient plutôt des femmes et leur statut HLAB27 était plus souvent négatif. Huit patients sans sacro-iliite présentaient une LI. La prévalence de la sacro-iliite et de la SpA chez les patients atteints de sarcoïdose a été estimée à 14,3\% (6/42) et $43 \%$ (6/14), respectivement, dans le sous-groupe LI. 
Sigaux et al. [12] ont publié récemment une étude prospective sur la lombalgie avec exploration IRM dans un groupe de 337 patients atteints de sarcoïdose confirmée par une biopsie. Sur les 64 patients présentant une lombalgie, il y avait 29 cas de LI. Les critères de diagnostic de la sacro-iliite étaient un œdème médullaire dans un compartiment de l'ASI sur une coupe pour les lésions bilatérales ou sur deux coupes consécutives lorsque la localisation était unilatérale. Deux lecteurs (un rhumatologue et un radiologue) ont analysé les IRM en aveugle et un consensus a été obtenu en cas de désaccord. Une sacro-iliite a été diagnostiquée chez 13 patients présentant une LI (5 avaient également une sacro-iliite radiographique selon les critères NYm). Les caractéristiques des patients du sous-groupe sacro-iliite n'ont pas été consignées. Une SpA a été diagnostiquée chez tous les patients présentant une sacro-iliite ; chez un patient souffrant de douleurs mécaniques, le diagnostic a été posé sur la base de la combinaison HLA-B27 positif et présence de psoriasis et d'enthésite et un patient présentait un signal vertébral inflammatoire, un statut HLA-B27 positif et des caractéristiques cliniques de SpA. La prévalence respective de la sacro-iliite et de la SpA a été estimée à 3,9 \% (13/337) et 4,5\% (15/337) dans le groupe sarcoïdose, 20,3\% (13/64) et 23,4 \% (15/64) dans le sous-groupe lombalgie et $44,8 \%(13 / 29)$ et $48,3 \%(14 / 29)$ dans le sous-groupe LI.

\section{b. Rapports de cas de sarcoïdose-sacro-iliite [4,13-42]}

Trente-et-un articles portant sur 35 cas au total ont été inclus dans l'analyse. Les données disponibles sur les caractéristiques de la lombalgie (critères de l'ASAS de lombalgie inflammatoire ou mécanique) étaient trop limitées pour être spécifiées. Les critères de l'ASAS pour la SpA ont été appliqués rétrospectivement. Une lombalgie présente depuis au moins trois mois et apparue avant 45 ans a été considérée comme inflammatoire, excepté lorsqu'elle était explicitement qualifiée de mécanique. La sacro-iliite a été diagnostiquée sur les IRM par la présence d'un œdème médullaire ou sur une radiographie pelvienne (NYm stade 2 pour les atteintes bilatérales, stade 3 ou 4 pour les atteintes unilatérales). Si les critères de l'ASAS n'étaient toujours pas remplis, d'autres caractéristiques cliniques et la positivité HLA-B27 ont également été prises en considération pour le diagnostic de SpA. Les principales caractéristiques sont résumées dans les tableaux 2 et 3 . L'évaluation des ASI a généralement été effectuée sur une radiographie pelvienne standard. Quatre articles n'indiquaient pas les modalités d'imagerie utilisées pour diagnostiquer la sacro-iliite. À la radiographie, six patients présentaient une sacro-iliite bilatérale de stade 2 , neuf patients présentaient une sacro-iliite unilatérale ou bilatérale de stade 3 et six patients présentaient une ankylose sacro-iliaque (stade 4). Deux rapports de cas ont considéré que l'imagerie des ASI était normale $[22,38]$ mais ont été inclus en raison du diagnostic de SpA établi par les auteurs. Un granulome confirmé par biopsie ou un syndrome de Löfgren a été rapporté dans tous les cas de sarcoïdose à l'exception du cas $n^{\circ} 2$ de Kremer et al. [22] dont la présentation clinique suggérait fortement une sarcoïdose sans granulome sur la biopsie de la carène.

\section{c. Atteinte granulomateuse des os iliaques [6,43-48]}

Nous avons classé 11 articles dans ce sous-groupe caractérisé par la présence de tissu granulomateux sur la biopsie des os iliaques ou par une imagerie suggérant une atteinte osseuse. Dans quatre articles toutefois, la sarcoïdose n'a pas été diagnostiquée selon les critères WASOG/ATS 
[annexe A, Doc. S1 : voir le matériel complémentaire accompagnant la version en ligne de cet article] en raison de la présence simultanée d'une infection mycobactérienne dans les prélèvements osseux ou pulmonaires. Cinq cas de sarcoïdose étaient accompagnés d'un granulome révélé par une biopsie des os iliaques. Pour les deux cas restants, Benelhadj et al. [46] suspectaient une lésion granulomateuse du fait d'une hyperactivité observée dans l'ASI gauche sur la scintigraphie au Tc ${ }^{99}$ avec un îlot osseux sur la TDM chez un patient présentant une atteinte osseuse périphérique, et Mijiyawa et al. [44] ont montré un granulome sur une biopsie osseuse de T10 et des changements ostéosclérotiques de l'os iliaque chez un patient présentant une sarcoïdose multiviscérale. En dehors de la radiographie, aucun signe de véritables dommages au niveau des ASI n'a été révélé dans ce sous-groupe sur les clichés TDM [48] et IRM [6]. Les principales caractéristiques des sept cas confirmés ou très probables sont résumées dans le tableau 4. Tous les patients souffraient de lombalgie. II y avait six femmes et un homme, d'âge médian 42,0 ans (IQR $\pm 12,0)$. L'atteinte iliaque unilatérale révélée par l'imagerie concernait six patients sur sept et était plus fréquente dans le groupe présentant une atteinte osseuse que la sacro-iliite unilatérale dans le groupe sarcoïdose-sacro-iliite. Une maladie granulomateuse touchant plusieurs organes était également fréquente. Le statut HLA-B27 n'était mentionné dans aucun article.

\section{Discussion}

L'arthropathie sacro-iliaque peut être associée à la sarcoïdose, mais la question de savoir s'il s'agit d'une caractéristique de la maladie n'est pas tranchée. La plupart des publications ont étudié l'association de la sarcoïdose avec la SpA et les patients présentant une LI étaient le plus souvent recrutés dans un service de rhumatologie. De plus, toutes les études transversales ont analysé la lombalgie et la sacro-iliite dans un groupe où la sarcoïdose était préexistante, tandis que la plupart des rapports de cas mentionnaient un diagnostic de sarcoïdose postérieur à l'apparition des symptômes de sacro-iliite dans près de la moitié des cas. Quelques auteurs ont observé une corrélation entre SpA et sarcoïdose, par exemple récemment dans la population générale taïwanaise [49] où la prévalence de spondylarthrite ankylosante a été estimée à 3,64 \% dans le groupe sarcoïdose contre $0,83 \%$ chez les témoins (odds ratio ajusté 3,80 [2,42-5,97]). Cette prévalence dans le groupe sarcoïdose est proche de celle rapportée par Sigaux et al. [12]. Le long délai moyen de 10,4 ans $( \pm 7,1)$ entre les premiers symptômes sacro-iliaques et le diagnostic de sarcoïdose pourrait également soutenir l'hypothèse que les deux maladies sont distinctes. 
L'atteinte sacro-iliaque pourrait également être imputable à une lésion de sarcoïdose sur les os iliaques ou du sacrum [6,45]. Depuis la première description d'une atteinte des os sacro-iliaques en 1951 [4], les critères de diagnostic de la sarcoïdose ont évolué et incluent désormais la confirmation histologique d'un granulome sarcoïdien et l'exclusion d'autres maladies granulomateuses. Les progrès récents de l'identification bactériologique avec de nouvelles techniques de culture et moléculaires, comme les tests PCR pour les mycobactéries, pourraient expliquer certains cas de diagnostic incorrect de sarcoïdose en présence de granulome dans des articles anciens [15] (annexe A, matériel complémentaire S1). Quoi qu'il en soit, la prévalence de la sarcoïdose osseuse (axiale et périphérique) peut fréquemment être sous-estimée puisque la moitié des cas sont asymptomatiques [3]. Ben Hassin et al. ont récemment jeté un nouvel éclairage sur la sarcoïdose osseuse en étudiant une cohorte multicentrique rétrospective de 88 patients [3] : des lésions osseuses ont été diagnostiquées à la présentation initiale de la sarcoïdose chez $39 \%$ des patients, avec un délai médian de 2,8 ans entre le diagnostic de sarcoïdose et l'atteinte osseuse. Le pelvis était touché dans $63 \%$ des cas de sarcoïdose osseuse axiale, ce qui représente $44 \%$ des localisations osseuses. Les taux d'atteinte des ganglions médiastinaux et extramédiastinaux, d'atteinte pulmonaire, de manifestations cutanées et d'hypercalcémie étaient significativement plus élevés chez les patients présentant des lésions osseuses. En outre, $52 \%$ des patients présentaient des symptômes osseux et $33 \%$ d'entre eux souffraient de douleurs dorsales ou pelviennes. Le traitement de la sarcoïdose osseuse reposait essentiellement sur des glucocorticoïdes. Cet article a décrit une atteinte granulomateuse dans les os pelviens qui, bien que rare, peut survenir plusieurs années après le premier diagnostic et peut avoir une présentation clinique similaire à la SpA en ce qui concerne la lombalgie. L'imagerie, IRM de préférence à TDM, peut faciliter la distinction entre atteinte osseuse et sacro-iliite. La biopsie osseuse semble donc indispensable lorsque la radiographie, l'IRM ou la TDM ne permet pas de déterminer avec certitude la cause des problèmes osseux (infection, métastases ou hémopathie).

En théorie, la sarcoïdose pourrait se manifester comme une synovite sacro-iliaque granulomateuse similaire à une synovite granulomateuse périphérique chronique. Elle peut toutefois être difficile à distinguer d'une synovite sacro-iliaque due à la SpA sans une analyse histologique. À ce jour, aucun cas de synovite granulomateuse des ASI n'a été rapporté. Des voies physiopathologiques communes ont cependant été décrites dans la $\mathrm{SpA}$ et la sarcoïdose. En réponse à la découverte de grandes quantités de TNFa dans les ASI des patients atteints de SpA, l'efficacité des traitements antiTNFa a été largement démontrée dans cette maladie. Les TNFa sont également impliqués dans la formation de granulome et l'efficacité de l'infliximab a été établie dans la sarcoïdose [50]. Parmi les articles étudiés dans cette revue, l'anti-TNFa infliximab a été utilisé pour traiter les deux maladies dans quatre cas $[32-34,42]$ avec de bons résultats, tandis que l'administration d'étanercept pour traiter la SpA a aggravé la sarcoïdose dans un autre [30]. Cette aggravation paradoxale sous anti-TNFa est aujourd'hui bien connue [9] et l'amélioration de la réaction granulomateuse à l'arrêt du traitement, de même que la prévalence des RMTS associées aux anti-TNFa plus élevée que dans la sarcoïdose ellemême [9] suggèrent que les deux maladies sont distinctes. L'IL-17 produite par les lymphocytes auxiliaires T 17 (Th17) constitue également une cible thérapeutique dans la SpA, et les anticorps monoclonaux anti-IL-17A tels que le sécukinumab sont efficaces [51]. II a aussi été démontré que le taux de lymphocytes Th17 augmente dans les granulomes, de même que celui des lymphocytes $T$ mémoires circulants chez les patients atteints de sarcoïdose [52], suggérant un rôle de ces lymphocytes dans la pathogenèse de la sarcoïdose. Par ailleurs, un nombre élevé de cellules productrices d'IL-17 a 
été associé à un meilleur résultat dans le syndrome de Löfgren [53] et des études complémentaires sont nécessaires pour déterminer l'intérêt de cibler l'IL-17A dans la sarcoïdose.

En ce qui concerne l'imagerie de la sacro-iliite, Sigaux et al. [12] ont diagnostiqué la majorité des cas par IRM dont seulement $38 \%$ (5/13) avaient également une sacroiliite radiographique. Le diagnostic de sacro-iliite par IRM et par radiographie concernait respectivement $45,3 \%$ et $17,2 \%$ des patients présentant une LI, $20,3 \%$ et $7,8 \%$ des patients souffrant de lombalgie et $3,9 \%$ et $1,5 \%$ de l'ensemble des 337 patients. Erb et al. [11] ont utilisé uniquement la radiographie et observé une prévalence similaire de $12,9 \%$ dans le sous-groupe $\mathrm{LI}$ et $8,2 \%$ dans le sous-groupe lombalgie. Kobak et al. [5] ont rapporté une prévalence de sacro-iliite radiographique de $43 \%$ dans le sous-groupe $\mathrm{LI}$ et de $14,3 \%$ dans le groupe sarcoïdose complet. Ces écarts s'expliquent par les différences entre les groupes étudiés. L'âge moyen des patients des études était similaire, mais la durée de la sarcoïdose était variable : elle était plus courte dans l'étude de Kobak et al. [5] (17,8 mois en moyenne) que dans celles d'Erb et al. [11] et de Sigaux et al. [12] (9,0 et 12,2 ans en moyenne, respectivement). Plus important, la durée de la lombalgie n'a été mentionnée que dans l'étude de Sigaux et al. [12], en moyenne de 3,6 $\pm 5,6$ ans dans le groupe LI. Compte tenu du délai moyen de neuf ans entre le l'apparition des symptômes sacro-iliaques et le diagnostic de spondylarthrite ankylosante[54], il aurait été intéressant de connaître la durée d'évolution de la lombalgie dans les deux autres études. Enfin, les groupes étudiés n'étaient pas homogènes, notamment en termes de caractéristiques géographiques, ce qui a pu influencer l'aspect génétique et la fréquence de la positivité du HLA-B27.

D'autres phénomènes peuvent expliquer la différence du nombre de cas de sacro-iliite entre ces études. La faible reproductibilité interlecteur et la sensibilité médiocre de la radiographie pour diagnostiquer la sacro-iliite sont bien connues [55]. La radiographie ne fournit qu'une vue en deux dimensions de l'ASI, ce qui peut conduire à diagnostiquer à tort des changements dégénératifs dans le compartiment ligamentaire antéro-inférieur de l'ASI comme une sacro-iliite, en particulier dans les cas bilatéraux de stade 2 et dans les cas d'érosions [55]. De même, la sclérose a été associée au sexe féminin [56] et pourrait expliquer la prédominance de négativité du HLA-B27 chez les femmes dans les études de Kobak et al. [5] et d'Erb et al. [11], qui ont utilisé la radiographie. De plus, I'IRM a une bonne spécificité mais aussi un taux élevé de faux positifs pour le diagnostic de la sacro-iliite car la présence d'un œedème médullaire (OM) peut conduire à surestimer les cas de sacro-iliite [57]. En outre, la présence d'un OM au niveau de l'ASI n'a pas été corrélée à des critères de LI tels que ceux de l'ASAS 2009 ou de Calin dans un groupe de patients souffrant de lombalgie.La raideur matinale $>60$ minutes était la seule caractéristique de douleur associée à l'OM [58]. Dans notre groupe sarcoïdose-sacro-iliite, les données des articles étudiés n'étaient pas suffisamment détaillées pour évaluer les caractéristiques de la lombalgie telles que la raideur matinale. En revanche, l'intensité de l'hypersignal en séquence STIR pourrait permettre de distinguer plus facilement les changements inflammatoires (avec un score moyen à élevé pour l'OM) des changements dégénératifs (avec un faible score pour l'OM) corrélés à l'âge mais pas à la LI et ni aux caractéristiques de SpA selon les critères de l'ASAS [56]. Sigaux et al. [12] n'ont pas effectué d'évaluation IRM systématique des ASI dans les cas de lombalgie non inflammatoire (évaluation réalisée chez seulement $9 / 35$ patients), ce qui peut avoir conduit à sousestimer le nombre de cas de sacro-iliite et de SpA selon les critères de l'ASAS. Cela étant, leur étude était la seule évaluant les caractéristiques de la SpA chez des patients souffrant de lombalgie mécanique. Enfin, un OM asymptomatique remplissant les critères de l'ASAS 2009 pour la sacro-iliite peut être présent chez $17,2 \%$ des individus en bonne santé ou après un stress physique des ASI dû par exemple au sport, à un entraînement militaire ou à un accouchement [57], et aucune évaluation des 
ASI par imagerie, y compris par IRM, n'a été décrite dans la littérature à ce jour chez des patients asymptomatiques atteints de sarcoïdose.

Dans cette revue systématique, une évaluation TDM des ASI n'a été réalisée que chez quelques patients dans les rapports de cas et chez aucun patient dans les études de cohorte. La TDM peut toutefois être intéressante pour évaluer l'anatomie des ASI et suivre les changements structuraux qui sont fréquents dans la population générale (environ $29 \%$ chez les 50-59 ans [59]). Une étude TDM des érosions serait également utile dans le diagnostic de la sacro-iliite [60], avec une forte corrélation au statut HLA-B27 et à la réponse aux AINS sur les images IRM dans les cas de LI [58]. Enfin, cette revue de la littérature interroge sur les critères de diagnostic de l'ASAS 2009 pour la SpA et la précision de l'imagerie radiographique, IRM et TDM en présence de caractéristiques telles que LI ou positivité du HLA-B27 chez les patients atteints de sarcoïdose.

L'un des résultats de cette revue systématique est l'identification de deux groupes de patients atteints de sarcoïdose avec atteinte sacro-iliaque: un groupe sarcoïdose-sacro-iliite qui inclut les patients atteints de sarcoïdose remplissant les critères de l'ASAS 2009 pour la SpA avec une prédominance de positivité du HLA-B27 et de LI d'une part, et un groupe de patients présentant une atteinte granulomateuse des os iliaques apparemment non associée à la SpA. La distinction entre ces deux présentations est importante en termes de traitement et d'évolution. La SpA est une maladie chronique nécessitant un suivi au long cours et un traitement par AINS ou anti-TNFa. L'atteinte osseuse requiert le plus souvent une corticothérapie qui donne de bons résultats même s'il s'agit parfois d'une manifestation d'une maladie systémique réfractaire nécessitant d'autres traitements immunosuppresseurs [3].

\section{Conclusion}

Les changements dans les ASI dus à la sarcoïdose incluent une sacro-iliite associée à la SpA et une atteinte osseuse granulomateuse. L'arthropathie sacro-iliaque de la sarcoïdose continue de faire l'objet de discussions. En présence de lombalgie, des symptômes inflammatoires devraient faire suspecter une SpA. Une radiographie pourrait être effectuée en premier lieu, mais en raison de la prévalence potentiellement supérieure de SpA dans la sarcoïdose et de plusieurs cas de sarcoïdose osseuse axiale, une IRM pelvienne et rachidienne doit être effectuée en cas de lombalgie inexpliquée chez un patient atteint de sarcoïdose. En cas d'atteinte osseuse suspectée, une biopsie osseuse peut être envisagée. La complexité de l'imagerie des ASI en cas de lombalgie inflammatoire, mécanique ou même en l'absence de douleurs souligne la nécessité de nouvelles investigations des ASI dans la sarcoïdose, avec évaluation clinique et biologique, notamment la recherche du HLA-B27, et avec imagerie multimodale (IRM et TDM).

\section{Sources de financement}

Aucun soutien financier ni autre avantage provenant de sources commerciales n'a été fourni pour ce travail. 
Articles identifiés par la recherche dans les bases de données

PubMed, $n=319$

Cochrane, $n=37$
Articles supplémentaires provenant d'autres sources

$$
n=5
$$

Articles après retrait des doublons

$$
n=337
$$

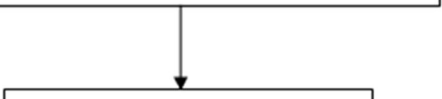

Examen des titres et des résumés

$$
n=337
$$

Articles exclus

$$
n=267
$$

Articles complets évalués pour déterminer l'éligibilité

$n=70$

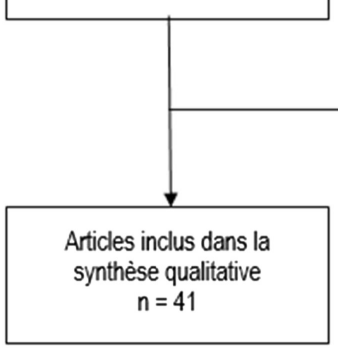

\section{Articles complets exclus}

$$
n=29
$$

- Réaction de type sarcoïdose après traitement anti-TNFa, $n=14$

Tuberculose possible, $n=4$

- Revue de la littérature, $n=2$

- Pas d'atteinte des ASI, $n=2$

- Études épidémiologiques, $n=2$

- Autre langue, $n=3$

- Pas d'antécédents de sarcoïdose, $n=1$

- $\quad$ Autre sujet, $n=1$ 


\section{Déclaration d'intérêts}

Les auteurs déclarent n'avoir aucun conflit d'intérêts.

\section{S1}

Annexe A. Matériel complémentaire

Du matériel complémentaire (Doc. S1) accompagnant cet article est disponible sur ... 


\section{Références}

[1] Valeyre D, Prasse A, Nunes H, Uzunhan Y, Brillet P-Y, Müller-Quernheim J. Sarcoidosis. Lancet 2014;383:1155-67. https://doi.org/10.1016/S0140-6736(13)60680-7.

[2] Goussault C, Albert J-D, Coiffier G, Lamer F, Guillin R, Le Goff B, et al. Ultrasound characterization of ankle involvement in Löfgren syndrome. Joint Bone Spine 2017. https://doi.org/10.1016/j.jbspin.2017.03.004.

[3] Ben Hassine I, Rein C, Comarmond C, Glanowski C, Saidenberg-Kermanac'h N, Meunier B, et al. Osseous sarcoidosis: A multicenter retrospective case-control study of 48 patients. Joint Bone Spine 2019;86:789-93. https://doi.org/10.1016/j.jbspin.2019.07.009.

[4] Verstraeten, J.M., Bekaert, J. Association de spondylite ankylosante et de sarcoidose. 1951;42:149-51.

[5] Kobak S, Sever F, Ince O, Orman M. The prevalence of sacroiliitis and spondyloarthritis in patients with sarcoidosis. Int J Rheumatol 2014;2014:289454. https://doi.org/10.1155/2014/289454.

[6] Binicier O, Sari I, Sen G, Onen F, Akkoc N, Manisali M, et al. Axial sarcoidosis mimicking radiographic sacroiliitis. Rheumatol Int 2009;29:343-5. https://doi.org/10.1007/s00296-008-06776.

[7] Moher D, Liberati A, Tetzlaff J, Altman DG, PRISMA Group. Preferred reporting items for systematic reviews and meta-analyses: the PRISMA statement. PLoS Med 2009;6:e1000097. https://doi.org/10.1371/journal.pmed.1000097.

[8] Costabel U, Hunninghake GW. ATS/ERS/WASOG statement on sarcoidosis. Sarcoidosis Statement Committee. American Thoracic Society. European Respiratory Society. World Association for Sarcoidosis and Other Granulomatous Disorders. Eur Respir J 1999;14:735-7. https://doi.org/10.1034/j.1399-3003.1999.14d02.x.

[9] Chopra A, Nautiyal A, Kalkanis A, Judson MA. Drug-Induced Sarcoidosis-Like Reactions. Chest 2018;154:664-77. https://doi.org/10.1016/j.chest.2018.03.056.

[10] Rudwaleit M, van der Heijde D, Landewé R, Listing J, Akkoc N, Brandt J, et al. The development of Assessment of SpondyloArthritis international Society classification criteria for axial spondyloarthritis (part II): validation and final selection. Ann Rheum Dis 2009;68:777-83. https://doi.org/10.1136/ard.2009.108233.

[11] Erb N, Cushley MJ, Kassimos DG, Shave RM, Kitas GD. An assessment of back pain and the prevalence of sacroilitis in sarcoidosis. Chest 2005;127:192-6. https://doi.org/10.1378/chest.127.1.192.

[12] Sigaux J, Semerano L, Nasrallah T, Nunes H, Bouvry D, Valeyre D, et al. High prevalence of spondyloarthritis in sarcoidosis patients with chronic back pain. Seminars in Arthritis and Rheumatism 2019. https://doi.org/10.1016/j.semarthrit.2019.03.006.

[13] Deshayes P, Desseauve J., Hubert J., Lemercier J.P., Geoffroy Y. Un cas de polyarthrite au cours d'une sarcoidose: Un cas de spondylarthrite ankylosante au cours d'une sarcoidose. Rev Rhum 1965;32:671-4.

[14] Ranfaing J, Banzet ML, Ledoux A, Couetdic G, Clement B. [Letter: Association of sarcoidosis, ankylosing spondylitis and monoclonal immunoglobulin]. Nouv Presse Med 1976;5:1006.

[15] Blanchon P, Paillas J, Lauriat H, Tominez G. [Pelvi-spinal and spinal localizations of BesnierBoeck-Schaumann's sarcoidosis]. Ann Med Interne (Paris) 1976;127:843-8.

[16] Gerster JC, Chappuis P. [Association of an acute sarcoidosis and ankylosing spondylitis in a patient (author's transl)]. Schweiz Rundsch Med Prax 1981;70:2356-9.

[17] Velasco Domínguez E, García Velasco F, Arranz Velasco JL, Mateos Otero J, Ergueta Martín P, López Palma LM. [Vertebral sarcoidosis with ankylosing spondyloarthritis and atlanto-axial luxation]. Rev Clin Esp 1983;168:423-6. 
[18] Kchir MM, Mazigh R, Charrad R, Boujnah MR, Chérif W, Baklouti N, et al. [Sacro-iliac involvement in sarcoidosis. Apropos of a case]. Tunis Med 1986;64:961-4.

[19] Kirkham B, Jobanputra P. Sarcoidosis and spondarthritis. Br J Rheumatol 1988;27:241.

[20] Stucki G, von Felten A, Speich R, Michel BA. Ankylosing spondylitis and sarcoidosis--coincidence or association? Case report and review of the literature. Clin Rheumatol 1992;11:436-9.

[21] Kötter I, Dürk H, Saal JG. Sacroiliitis in sarcoidosis: case reports and review of the literature. Clin Rheumatol 1995;14:695-700.

[22] Kremer P, Gallinet E, Benmansour A, Despaux J, Toussirot E, Wendling D. Sarcoidosis and spondylarthropathy. Three case-reports. Rev Rhum Engl Ed 1996;63:405-11.

[23] Alijotas-Reig J, Panisello-Royo J. Acute sacroiliac arthritis and sarcoidosis. Rev Rhum Engl Ed 1997;64:278.

[24] Garazi S, Roux S, Palazzo E, Meyer O. [Sarcoidosis and spondyloarthropathy. Apropos of a case]. Ann Med Interne (Paris) 1998;149:303-4.

[25] Abouzahir A, El Maghraoui A, Tabache F, Bezza A, Chaari J, Ghafir D, et al. [Sarcoidosis and ankylosing spondylitis. A case report and review of the literature]. Ann Med Interne (Paris) 2002;153:407-10.

[26] Gómez-Puerta JA, Musuruana J, Saez C, Cervera R, Font J. [Sarcoidosis as seronegative spondyloarthropathy]. Biomedica 2005;25:435-8.

[27] Alaoui F-Z, Talaoui M, Benamour S. [Osteo-articular manifestations of sarcoidosis]. Presse Med 2005;34:19-24.

[28] Vandergheynst F, Tant L. Sarcoidosis masquerading as ankylosing spondylitis. Eur J Intern Med 2006;17:74. https://doi.org/10.1016/j.ejim.2005.09.014.

[29] Sezer I, Melikoglu MA, Cay HF, Kocabas H, Kacar C. A co-occurrence of sarcoidosis and ankylosing spondylitis: a case report. Rheumatol Int 2008;28:605-7. https://doi.org/10.1007/s00296-007-0483-6.

[30] Louie GH, Chitkara P, Ward MM. Relapse of sarcoidosis upon treatment with etanercept. Ann Rheum Dis 2008;67:896-8. https://doi.org/10.1136/ard.2007.078840.

[31] Levy S, Sandhu V. Ankylosing spondylitis and pulmonary sarcoidosis--a case report and discussion of the literature. Rheumatology (Oxford) 2008;47:1733-4. https://doi.org/10.1093/rheumatology/ken366.

[32] Agrawal S, Bhagat S, Dasgupta B. Sarcoid sacroiliitis: successful treatment with infliximab. Ann Rheum Dis 2009;68:283. https://doi.org/10.1136/ard.2007.087155.

[33] Ochi S, Nanki T, Kaneko H, Honne K, Miyazaki Y, Komano Y, et al. Successful treatment of ankylosing spondylitis coexisting with pulmonary sarcoidosis by infliximab. Clin Exp Rheumatol 2009;27:698-9.

[34] Malaviya AN, Sawhney S, Kapoor S, Garg S. Vertebral sarcoid mimicking ankylosing spondylitis or just a co-incidence? J Assoc Physicians India 2010;58:709-11.

[35] Gürer G, Unubol Al, Bozbaş3 GT. A Case of Sacroilitis Developing After Löfgren's Syndrome. Arch Rheumatol 2011;26:265-8. https://doi.org/10.5152/tjr.2011.043.

[36] El Ouazzani FZ, Tahiri L, Akasbi N, Kadi N, Harzy T. Sarcoidose et spondylarthrite ankylosante: une association rare. Pan Afr Med J 2011;8.

[37] Briongos-Figuero LS, Ruiz-de-Temiño Á, Pérez-Castrillón JL. Sarcoidosis and sacroiliitis, a case report. Rheumatol Int 2012;32:2949-50. https://doi.org/10.1007/s00296-011-2100-y.

[38] Kara M, Özçakar L, Kalyoncu F, Akinci2 A. Early Undifferentiated Spondyloarthropathy in a Patient with Sarcoidosis. Arch Rheumatol 2013;28:206-8. https://doi.org/10.5606/tjr.2013.3000.

[39] Kobak S, Sever F, Sivrikoz O, Karaarslan A. Coexistence of Ankylosing Spondylitis and Löfgren's Syndrome. Case Reports in Rheumatology 2014;2014. https://doi.org/10.1155/2014/747698.

[40] Kobak S, Karaarslan A, Aycan H. Unilateral lymphedema as first presentation of sarcoidosis. Reumatol Clin 2016;12:59-60. https://doi.org/10.1016/j.reuma.2015.03.014. 
[41] Wafa H, Saoussen M, Dhia K, Imen Z, Montacer KM. Sarcoidosis and spondyloarthritis: A coincidence or common etiopathogenesis? Caspian J Intern Med 2017:9:100-3. https://doi.org/10.22088/cjim.9.1.100.

[42] Rahul CD, Sharma RK, Talwar D. Sarcoidosis with ankylosing spondylitis: changing therapeutic landscape. 1 2018;35:285-8.

[43] Brun J, Pozzetto H, Buffat JJ, Soustelle J, Vauzelle JL, Patin R. [Vertebral and sacroiliac sarcoidosis with image of Pott's pseudo-abscess. Cure by corticotherapy]. Presse Med 1966;74:511-6.

[44] Mijiyawa M, Fereres M, Deutsch JP, Awada H, Dougados M, Amor B. [Pelvi-rachidian involvement in sarcoidosis. Apropos of a case. Review of the literature]. Rev Rhum Mal Osteoartic 1989;56:529-32.

[45] Golzarian J, Matos C, Golstein M, Stallenberg B, Depierreux M, Struyven J. Case report: Osteosclerotic sarcoidosis of spine and pelvis: plain film and magnetic resonance imaging findings. Br J Radiol 1994;67:401-4. https://doi.org/10.1259/0007-1285-67-796-401.

[46] Benelhadj S, Patrois F, Duet M, Bérolatti B, Mundler O. Radioisotope bone scanning in chronic osseous sarcoidosis. Clin Nucl Med 1996;21:371-4.

[47] Franco M, Passeron C, Tieulie N, Verdier JF, Benisvy D. Long-term radiographic follow-up in a patient with osteosclerotic sarcoidosis of the spine and pelvis. Rev Rhum Engl Ed 1998;65:58690.

[48] Andres E, Loth F, Orion B, Marcellin L, Durckel J. lliac bone defects revealing systemic sarcoidosis. Joint Bone Spine 2001;68:74-5.

[49] Wu C-H, Chung P-I, Wu C-Y, Chen Y-T, Chiu Y-W, Chang Y-T, et al. Comorbid autoimmune diseases in patients with sarcoidosis: A nationwide case-control study in Taiwan. J Dermatol 2017;44:423-30. https://doi.org/10.1111/1346-8138.13654.

[50] Heidelberger V, Ingen-Housz-Oro S, Marquet A, Mahevas M, Bessis D, Bouillet L, et al. Efficacy and Tolerance of Anti-Tumor Necrosis Factor a Agents in Cutaneous Sarcoidosis: A French Study of 46 Cases. JAMA Dermatol 2017;153:681-5. https://doi.org/10.1001/jamadermatol.2017.1162.

[51] Baeten D, Sieper J, Braun J, Baraliakos X, Dougados M, Emery P, et al. Secukinumab, an Interleukin-17A Inhibitor, in Ankylosing Spondylitis. N Engl J Med 2015;373:2534-48. https://doi.org/10.1056/NEJMoa1505066.

[52] Ten Berge B, Paats MS, Bergen IM, van den Blink B, Hoogsteden HC, Lambrecht BN, et al. Increased IL-17A expression in granulomas and in circulating memory T cells in sarcoidosis. Rheumatology (Oxford) 2012;51:37-46. https://doi.org/10.1093/rheumatology/ker316.

[53] Ostadkarampour M, Eklund A, Moller D, Glader P, Olgart Höglund C, Lindén A, et al. Higher levels of interleukin IL-17 and antigen-specific IL-17 responses in pulmonary sarcoidosis patients with Löfgren's syndrome. Clin Exp Immunol 2014;178:342-52. https://doi.org/10.1111/cei.12403.

[54] Mau W, Zeidler H, Mau R, Majewski A, Freyschmidt J, Stangel W, et al. Clinical features and prognosis of patients with possible ankylosing spondylitis. Results of a 10-year followup. J Rheumatol 1988;15:1109-14.

[55] Christiansen AA, Hendricks O, Kuettel D, Hørslev-Petersen K, Jurik AG, Nielsen S, et al. Limited Reliability of Radiographic Assessment of Sacroiliac Joints in Patients with Suspected Early Spondyloarthritis. J Rheumatol 2017;44:70-7. https://doi.org/10.3899/jrheum.160079.

[56] Arnbak B, Grethe Jurik A, Hørslev-Petersen K, Hendricks O, Hermansen LT, Loft AG, et al. Associations Between Spondyloarthritis Features and Magnetic Resonance Imaging Findings: A Cross-Sectional Analysis of 1,020 Patients With Persistent Low Back Pain. Arthritis \& Rheumatology (Hoboken, NJ) 2016;68:892-900. https://doi.org/10.1002/art.39551.

[57] Baraliakos X, Richter A, Feldmann D, Ott A, Buelow R, Schmidt CO, et al. Frequency of MRI changes suggestive of axial spondyloarthritis in the axial skeleton in a large population-based cohort of individuals aged <45 years. Ann Rheum Dis 2020;79:186-92.

https://doi.org/10.1136/annrheumdis-2019-215553. 
[58] Arnbak B, Jurik AG, Jensen TS, Manniche C. Association Between Inflammatory Back Pain Characteristics and Magnetic Resonance Imaging Findings in the Spine and Sacroiliac Joints. Arthritis Care Res (Hoboken) 2018;70:244-51. https://doi.org/10.1002/acr.23259.

[59] Eno J-JT, Boone CR, Bellino MJ, Bishop JA. The prevalence of sacroiliac joint degeneration in asymptomatic adults. J Bone Joint Surg Am 2015;97:932-6. https://doi.org/10.2106/JBJS.N.01101.

[60] Chan J, Sari I, Salonen D, Inman RD, Haroon N. Development of a Screening Tool for the Identification of Sacroiliitis in Computed Tomography Scans of the Abdomen. J Rheumatol 2016;43:1687-94. https://doi.org/10.3899/jrheum.150939. 